SERIE B - INFORMATIK

\title{
Isoperimetric Inequalities for Densities of Lattice-periodic Sets
}

\author{
Peter Braß* \\ B 97-05 \\ June 1997
}

\begin{abstract}
The minimum boundary length density of a lattice-periodic set with given period-lattice and area density is determined, together with the extremal sets, and a conjecture on the higher-dimensional analogue is made. This improves previous results of Hadwiger for $d$-dimensional sets with integer period lattice and of Schnell and Wills for twodimensional sets with arbitrary period lattice.
\end{abstract}

* Institut für Informatik, Freie Universität Berlin, Takustraße 9, D-14195 Berlin, Germany email: brass@inf.fu-berlin.de 


\title{
Isoperimetric Inequalities for Densities \\ of Lattice-periodic Sets
}

by

\author{
Peter Brass, Berlin
}

\begin{abstract}
The minimum boundary length density of a lattice-periodic set with given period lattice and area density is determined, together with the extremal sets, and a conjecture on the higher-dimensional analogue is made. This improves previous results of Hadwiger for $Z^{d}$-periodic $d$-dimensional sets and of Schnell and Wills on twodimensional sets with arbitrary period-lattice.
\end{abstract}

\section{Introduction}

A set $X \subset \mathbb{R}^{d}$ is lattice-periodic with period lattice $\Gamma$ iff the lattice translations from $\Gamma$ are symmetries of $X$, that is $X+\gamma=X$ for each $\gamma \in \Gamma$. Figure 1 shows a periodic set and the fundamental parallelograms of its period lattice.

Any nonempty such set $X$ is necessarily unbounded, but if it is reasonably wellformed (e.g. is locally the limit of polyhedral approximations) we can define its volume density $v(X)$ and perimeter density $p(X)$ as the average amounts of $X$ and $\partial X$ per unit volume of $\mathbb{R}^{d}$. The isoperimetric problem for lattice-periodic sets then is to determine the minimum perimeter density of a set with given period lattice and volume density. Since we may exchange $X$ and its complement without changing the perimeter density, the minimum perimeter density is the same for the volume densities $v$ and $1-v$.

This problem was first studied by HADWIGER [1] for the case of $Z^{d}$-periodic sets; he proved the lower bound $p(X) \geq 8 v(X)(1-v(X))$, which remarkably holds independent of the dimension $d$. This bound is sharp only for $v(X) \in\left\{0, \frac{1}{2}, 1\right\}$ and holds only for the special period lattice $Z^{d}$.



Figure 1.

1991 Mathematics Subject Classification: 51M16

Key words: isoperimetric inequalities, periodic sets 
In the two-dimensional case SCHNELL and WiLls [4] extended this bound to arbitrary period lattices $\Gamma \subset \mathbb{R}^{2}$, proving

$$
p(X) \geq 8 \frac{\min \{\|\gamma\| \mid \gamma \neq 0, \gamma \in \Gamma\}}{\operatorname{det}(\Gamma)} v(X)(1-v(X))
$$

In this two-dimensional case $p(X)$ denotes the boundary length density and $v(X)$ the area density of $X$. Again the bound is sharp only for $v(X) \in\left\{0, \frac{1}{2}, 1\right\}$. For higher dimensions $d$ and arbitrary period lattices $\Gamma \subset \mathbb{R}^{d}$ Schnell [3] proved

$$
p(X) \geq 2 d^{-\frac{3}{2}} \frac{\min \left\{\operatorname{det}(\hat{\Gamma}) \mid \begin{array}{l}
\hat{\Gamma} \text { is a }(d-1) \text {-dimensional } \\
\text { sublattice of } \Gamma
\end{array}\right.}{\operatorname{det}(\Gamma)} v(X)(1-v(X)) .
$$

Using the last successive minimum $\lambda_{d}(\Gamma)$ of $\Gamma$ instead of the minimal subdeterminants Schnell [2] found the bound

$$
p(X) \geq \frac{8}{\operatorname{det}(\Gamma) \lambda_{d}(\Gamma)} v(X)(1-v(X))
$$

\section{Results and Conjectures}

Let $\Gamma$ denote a lattice in $\mathbb{R}^{d}$ and $X \subset \mathbb{R}^{d}$ a $\Gamma$-periodic set. If $X$ is well-formed in the sense that volume and surface area are defined for some finite section (e.g. with a ball) which is big enough to contain a fundamental parallelotope of $\Gamma$, then we can define volume and perimeter densities of $X$. For this we can use the limits of the intersection with large balls $v(X):=\lim _{r \rightarrow \infty} \frac{\operatorname{vol}\left(X \cap B_{r}\right)}{\operatorname{vol}\left(B_{r}\right)}$ and $p(X):=\lim _{r \rightarrow \infty} \frac{\operatorname{area}\left(X \cap B_{r}\right)}{\operatorname{vol}\left(B_{r}\right)}$. Equivalently we may select a fundamental parallelotope $P$ such that the intersection of the boundaries $\partial P \cap \partial X$ has $(d-1)$-dimensional measure zero and define $v(X):=\frac{\operatorname{vol}(X \cap P)}{\operatorname{vol}(P)}$ and $p(X):=\frac{\operatorname{area}(\partial X \cap P)}{\operatorname{vol}(P)}$. In the two-dimensional case considered in the theorem $v$ and $p$ denote the area- and boundary-length densities of $X$.

Theorem : Let $X \subset \mathbb{R}^{2}$ be a $\Gamma$-periodic set for which $v(X)$ and $p(X)$ are defined. Then

$$
p(X) \geq 2 \min \left(\sqrt{\frac{\pi}{\operatorname{det}(\Gamma)} v(X)}, \frac{\min \{\|\gamma\| \mid \gamma \neq 0, \gamma \in \Gamma\}}{\operatorname{det}(\Gamma)}, \sqrt{\frac{\pi}{\operatorname{det}(\Gamma)}(1-v(X))}\right)
$$

This lower bound is reached for each given $v$ by one of the following sets

1) $X=B_{r}+\Gamma$ with $r=\sqrt{\frac{\operatorname{det}(\Gamma)}{\pi} v}$, that is the union of circular discs, one per fundamental domain,

2) $X=\mathbb{R} \gamma+\{t \hat{\gamma} \mid t \in \mathbb{R},(t-\lfloor t\rfloor) \leq v\}$, where $\gamma$ is the minimum-norm nonzero vector of $\Gamma$ and $\hat{\gamma}$ another element of $\Gamma$ such that $\gamma, \hat{\gamma}$ generate a fundamental parallelogramm,

3) $X=\mathbb{R}^{2} \backslash\left(B_{r}+\Gamma\right)$ with $r=\sqrt{\frac{\operatorname{det}(\Gamma)}{\pi}(1-v)}$, that is the plane with circular holes, one per fundamental domain. 

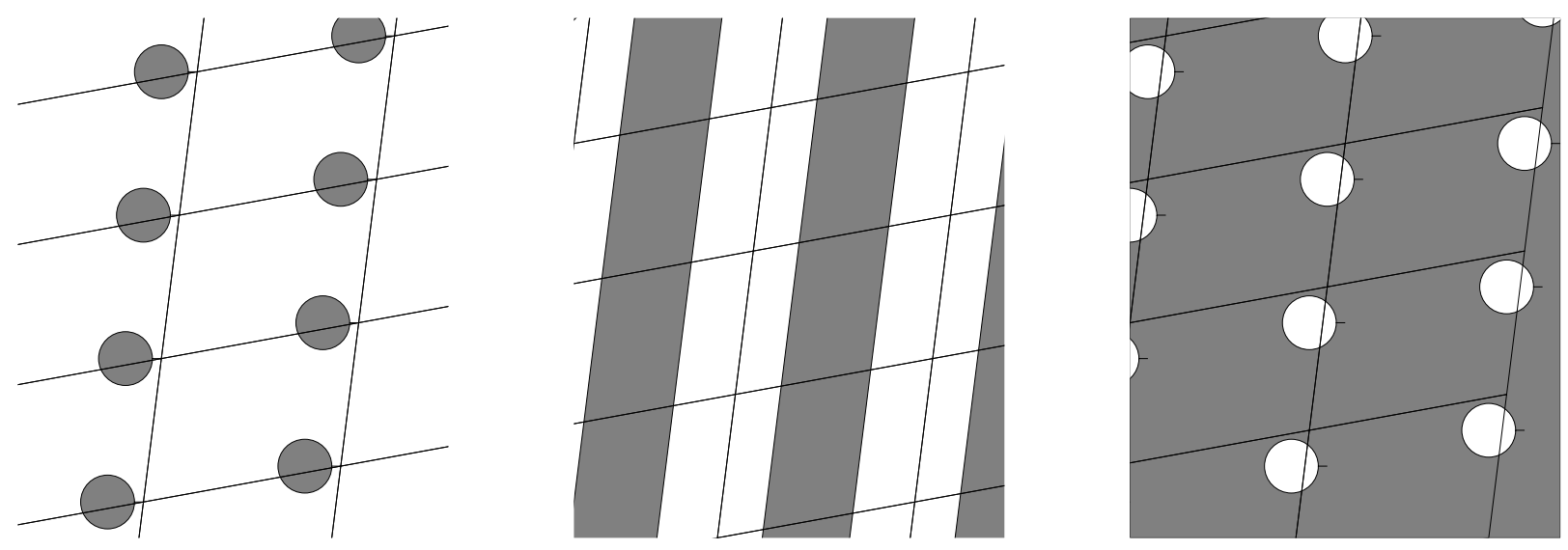

Figure 2.

We believe that this structure of the extremal sets holds also in higher dimensions. Using $\kappa_{i}$ for the volume of the $i$-dimensional ball of radius 1 and $\operatorname{det}_{i}(\Gamma)$ for the minimum determinants of $i$-dimensional sublattices of $\Gamma$ we can state the

Conjecture: Let $X \subset \mathbb{R}^{d}$ be a $\Gamma$-periodic set for which $v(X)$ and $p(X)$ are defined. Then

$$
p(X) \geq \min _{i=1, \ldots, d} i\left(\frac{\operatorname{det}_{d-i}(\Gamma) \kappa_{i}}{\operatorname{det}_{d}(\Gamma)}\right)^{\frac{1}{i}}(\min (v(X), 1-v(X)))^{1-\frac{1}{i}} .
$$

Probably the same structure holds even if the period lattice is not fulldimensional: The sets of minimal perimeter with given volume per fundamental domain consist of $i$-dimensional cylinders over $d-i$-dimensional balls, the cylinders taken in subspaces where the determinant of the sublattice is minimal, or the complement of such a set.

\section{Proof}

Let $X$ be a set with the given period lattice $\Gamma$ and volume density $v$ which is of minimum perimeter density among all such sets. We may assume $X$ to be closed. Let $\left\{X_{\iota}\right\}_{\iota \in I}$ be the connected components of $X=\bigcup_{\iota \in I} X_{\iota}$, and let $\Lambda\left(X_{\iota}\right)$ be the maximal sublattice of $\Gamma$ that leaves $X_{\iota}$ invariant $\left(X_{\iota}+\Lambda\left(X_{\iota}\right)=X_{\iota}\right)$.

If one of the $\Lambda\left(X_{\iota}\right)$ is nontrivial, i.e. there is a nonzero vector $t \in \Lambda\left(X_{\iota}\right)$, then $X_{\iota}$ contains with each point $p \in X_{\iota}$ the whole one-dimensional point-lattice $(p+z t)_{z \in Z \text {. }}$ Since $X_{\iota}$ is connected, there is a (shortest) arc $\alpha_{p}$ joining $p$ and $p+t$ in $X$, and this arc stays within a bounded distance to the line through $p$ and $p+t$. We can extend this arc periodically to a set $\bigcup_{z \in Z}\left(\alpha_{p}+z t\right) \subset X_{\iota}$ which is connected, contains all the lattice points $(p+z t)_{z \in Z}$, and stays within a bounded distance to the line $(p+r t)_{r \in \mathbb{R}}$. So there is a pseudoline $\alpha_{\iota} \subset X_{\iota}$ which stays within a bounded distance to the line $(p+r t)_{r \in \mathbb{R}}$ and which cuts the plane in two halves. If there is another connected component $X_{j}$ which also has nontrivial $\Lambda\left(X_{j}\right)$, then the associated lines may not intersect (being in distinct connected components); so each nonzero vector $s \in \Lambda\left(X_{j}\right)$ must be collinear with each nonzero vector $t \in \Lambda\left(X_{\iota}\right)$. 
Therefore either there is one $\Lambda\left(X_{\iota}\right)$ that is two-dimensional and all other $X_{j}$ have a trivial $\Lambda\left(X_{j}\right)$, or all nontrivial $\Lambda\left(X_{\iota}\right)$ generate the same 1-dimensional subspace (i.e. are collinear). Since $X$ is $\Gamma$-periodic, we have $\Gamma / \Lambda\left(X_{L}\right)$ copies of connected component $X_{\iota}$ in $X$. So if there is an $X_{\iota}$ with two-dimensional $\Lambda\left(X_{\iota}\right)$, we have $\Lambda\left(X_{\iota}\right)=\Gamma$, for otherwise there would be further connected components $X_{j}$ with twodimensional $\Lambda\left(X_{j}\right)$. And if $\Lambda\left(X_{\iota}\right)$ is one-dimensional, it must be generated by a primitive lattice vector of $\Gamma$, for otherwise there are several collinear copies of $X_{\iota}$, which contain alternating points of the same one-dimensional sublattice, and which are translates of each other, so they intersect.

So there are three possible cases: either all connected components have trivial $\Lambda$, or there are connected components with a one-dimensional $\Lambda$ which is generated by a primitive vector of $\Gamma$, or there is one component with $\Lambda=\Gamma$, and the complement consists of bounded sets with trivial $\Gamma$.

If there is a component $X_{\iota}$ with one-dimensional $\Lambda$ which is generated by $v \in \Gamma$, then $p(X) \geq 2 \frac{\|v\|}{\operatorname{det}(\Gamma)}$. For let $w \in \Gamma$ be a vector such that $\{v, w\}$ generates $\Gamma$ (this exists, since $v$ is primitive). Then $\left(z w+X_{\iota}\right)_{z \in \mathbf{Z}}$ are further connected components of $X$. For each $m, n \in \mathbb{N}$ the parallelogram $0, m v, m v+n w, n w$ is intersected by $n+O(1)$ copies of $X_{\iota}$, each of which (with the exception of the first and last $O(1)$ copies) has a boundary length of at least $2 m\|v\|$ within this parallelogram (which is of area $m n \operatorname{det}(\Gamma)$. So the density contributed by the translates of $X_{\iota}$ is at least $2 \frac{\|v\|}{\operatorname{det}(\Gamma)}$ for some nonzero $v \in \Gamma$. Since the set has minimal $p(X)$, it must be at least as good as the parallel strips construction of the theorem, so $v$ is a vector of minimum length, and there are no other connected components in $X$ (which could only increase $p(X)$ ).

So we may restrict us to the case that no $X_{\iota}$ has one-dimensional $\Lambda$. Exchanging $X$ and $\mathbb{R}^{2} \backslash X$ one sees that the other two cases are symmetric. Therefore we consider only the first case, i.e. all connected components have trivial $\Lambda$. Then we can partition the connected components into equivalence classes by $\Gamma$; we select one element $\left(\hat{X}_{\kappa}\right)_{\kappa \in K}$ of each equivalence class. Since there is one copy of each $\hat{X}_{\kappa}$ per each fundamental domain of $\Gamma$, we have $v(X)=\frac{1}{\operatorname{det}(\Gamma)} \sum_{\kappa \in K} \operatorname{vol}\left(\hat{X}_{\kappa}\right)$ and $p(X)=\frac{1}{\operatorname{det}(\Gamma)} \sum_{\kappa \in K} p\left(\hat{X}_{\kappa}\right)$. For each $\hat{X}_{\kappa}$ we may apply the standard isoperimetric inequality, giving $p\left(\hat{X}_{\kappa}\right) \geq 2 \sqrt{\pi \operatorname{vol}\left(\hat{X}_{\kappa}\right)}$, so we get

$$
p(X) \geq \frac{1}{\operatorname{det}(\Gamma)} \sum_{\kappa \in K} 2 \sqrt{\pi \operatorname{vol}\left(\hat{X}_{\kappa}\right)} \geq \frac{2}{\operatorname{det}(\Gamma)} \sqrt{\pi \sum_{\kappa \in K} \operatorname{vol}\left(\hat{X}_{\kappa}\right)}=2 \sqrt{\frac{\pi}{\operatorname{det}(\Gamma)} v(X)} .
$$

The same lower bound, with $1-v(X)$ instead of $v(X)$, holds in the last case (one $X_{\iota}$ with $\left.\Lambda\left(X_{\iota}\right)=\Gamma\right)$, in which we exchanged $X$ and $\mathbb{R}^{2} \backslash X$. The lower bound of the theorem is now the minimum of the three possibilities for the extremal sets. This proves the theorem.

\section{References}

[1] H. HADWIGER: Gitterperiodische Punktmengen und Isoperimetrie, Monatshefte für Mathematik 76 (1972), 410-418 
[2] U. Schnell: Lattice inequalities for convex bodies and arbitrary lattices, Monatshefte für Mathematik 116 (1993) 331-337.

[3] U. Schnell: Minimal determinants and lattice inequalities Bull. London Math. Soc. 24 (1992) 606-612

[4] U. Schnell and J. Wills: Two isoperimetric inequalities with lattice constraints, Monatshefte für Mathematik 112 (1991) 227-233 